\title{
Health inequalities in South-East Asia - extent, causes, and principles for policy action
}

\author{
Sayeeda Rahman', Md. Anwarul Azim Majumder
}

${ }^{1,2}$ Lecturer; Department of Clinical Sciences, School of Medical Sciences, University of Bradford, West Yorkshire, Bradford, UK.

The health of people in South-East Asia (SEA) region has improved markedly over the last 50 years. In 1960 life expectancy at birth for men was 44 years and 42.5 for women. ${ }^{1}$ By 1980 it was 55.4 and 55.3 years respectively. In 2010 the figures were 63.9 and 66.7. However, despite these huge improvements, there are marked health inequalities within and across countries in the SEA region. The gap in health outcomes between those at the top and bottom ends of the social scale remains large and in some countries continues to widen. ${ }^{2}$ Of 187 countries ranked on the basis of their level of development in the Human Development Report 2011, the countries of the SEA region are categorized within 'medium' or 'low' human development'. The average gross national income per capita (PPP int. \$) of the region is approximately one third of the global average and more people in the region (39\%) live on $<\$ 1$ (PPP int. \$) a day in comparison to the global population $(25.9 \%)$.

The SEA region shoulders a significant proportion of the global disease burden and lags behind most other regions in its overall health attainments. In the region, stillbirth rate, neonatal mortality rate, infant mortality rate, under-five mortality rate and adult mortality rate are higher than the global averages. ${ }^{1,4}$ These inequalities mean poorer health, reduced quality of life and early death for many people. Maternal and child health are still major concerns and are prominent in low socio-economic groups. ${ }^{4}$ Skilled birth attendance is considered as an important determinant of maternal mortality. In Bangladesh, the percentage of births attended by skilled health personnel is the lowest in the region and the difference is more prominent in rural areas, lowest wealth quintile and lowest educational level of mothers. ${ }^{4}$ The region has also relatively few health personnel and hospital beds than the global averages. The percent of GDP allocation on healthcare is lower in the region in comparison to other regions. The regional health spending accounted for $3.8 \%$ of GDP in 2008, below than the averages of low income countries $(5.4 \%)$, lower middle income countries $(4.3 \%)$ and global $(8.5 \%)$ averages. ${ }^{1}$ $\mathrm{WHO}^{5}$ recommended at least $5 \%$ of GDP allocation as the requirement for the universal coverage, however six SEA countries allocated less than $5 \%$ of GDP on health care.

Health inequality is defined as 'systematic differences in health status between different socio-economic groups' and which can be either absolute or relative. ${ }^{6}$ The determinants which affect health outcome of a population and lead to health inequality can be grouped under four broad headings: socioeconomic and political context, socioeconomic position, intermediary determinants and health systems factors. ${ }^{2}$ Socioeconomic-political context encompasses a wide range of structural, cultural and functional aspects of a social system. Socioeconomic position was measured by wealth, education and occupation. Intermediary determinants included living and working conditions and behavioural and biological factors. Access to and quality of health services were included as health systems factors.

There is a wealth of evidence to support the increase of extent and trends of health inequality and widening health gap within and across the SEA countries. Reducing health inequalities has a number of implications. ${ }^{2}$ Health equality is a central dimension of overall equity and justice, which indicates the capabilities of individuals and groups to participate in and benefit from social and economic development. Good health enables people to participate in socio-economic development which leads to better economic performance of the society. Health inequalities most adversely impact vulnerable and disadvantaged groups of the society, thereby further reducing their quality of life and their ability to contribute to overall social and economic development.

Within the countries of SEA region, there are many examples of systematic differences in health between different social groups. In the region, most disadvantaged groups have worse health and higher mortality. There is an urgent need to take appropriate action to reduce the widespread health inequalities and their root causes. Strategies and policy actions should be undertaken to alleviate inequalities in health by reviewing the social and economic determinants of population health, increasing the capacity of a country to invest more in health, and integrating these strategies and policies into the national agenda for development. The government should prioritise the need to tackle the causes and consequences of health inequalities as a part of its commitment to deliver economic prosperity and social justice. Long-term sustainable action

Correspondence: Dr. Sayeeda Rahman, Lecturer, Department of Clinical Sciences, School of Medical Sciences, University of Bradford, Bradford BD7 1DP, West Yorkshire, UK. E-mail: srahman6@bradford.ac.uk. 
to break the cycle of deprivation and its impact on avoidable ill health should be at the heart of national/regional policy development and implementation.

\section{References}

1. World Bank. How we Classify Countries. http://data.worldbank.org/about/countryclassifications (accessed 31 May 2012)

2. WHO. Health inequities in the South-East Asia Region: selected country case studies. New Delhi: World Health Organization, Regional Office for South-East Asia, 2009.

3. UNDP. Human Development Report 2011. Sustainability and Equity: A Better Future for All. New York: United Nations Development Programme, 2011.
4. Majumder MAA. World Health Statistics 2011: How does Bangladesh compare with other South-East Asian countries? South East Asia J Public Health 2011:1:3-8.

5. UNESCAP, Asian Development Bank (ADB), United Nations Development Programme (UNDP). Paths to 2015. MDG Priorities in Asia and the Pacific. AsiaPacific MDG Report 2010/11. Bangkok: United Nations, Economic and Social Commission for Asia and the Pacific, 2011.

6. Dahlgren G, Whitehead M. Levelling Up: Social inequalities in health concern systematic differences' in health status between different socioeconomic groups, Geneva: World Health Organization, 2007. 